\title{
Competition Equilibrium Analysis of China's Luxury Car Market Based on Three-Dimensional Grey Lotka-Volterra Model
}

\author{
Xiao-Lan Wu, Sheng-Yuan Wang (D), Ya-Zhen Liu, Jing Liang, and Xin Yu \\ Nanjing XiaoZhuang University, Nanjing, Jiangsu, China \\ Correspondence should be addressed to Sheng-Yuan Wang; 56439976@qq.com
}

Received 16 August 2021; Revised 30 November 2021; Accepted 14 December 2021; Published 23 December 2021

Academic Editor: Chittaranjan Hens

Copyright (C) 2021 Xiao-Lan Wu et al. This is an open access article distributed under the Creative Commons Attribution License, which permits unrestricted use, distribution, and reproduction in any medium, provided the original work is properly cited.

\begin{abstract}
An enterprise must be able to conduct in-depth analysis of the existing data as the information has certain grey characteristics, if it wants to occupy a dominant position in the fierce market competition. In this paper, a compound three-dimensional grey Lotka-Volterra model is developed to carry out the grey transformation of the original data, so that the data can have better simulation accuracy, and the observation noise of the original data can be reduced. The competitive situation analysis based on the three-dimensional grey Lotka-Volterra model can help enterprises better understand the market situation. This paper takes the luxury brand automobile market in mainland China as an example to conduct a competitive analysis and a balanced development simulation. It can be found that, based on Three Species System Analysis, there is a symbiotic relationship among automobile enterprises and that the three species model can be adopted in analyzing the competition and cooperation among enterprises. Through balanced development of a Symbiotic System Analysis, the results of symbiotic optimization under the achievable equilibrium state of three populations are obtained and they show that the proposed method can be used effectively to conduct the market competition analysis. It is thus of great importance to study the relationships among enterprises as it is helpful for enterprises to make strategic policies.
\end{abstract}

\section{Introduction}

After the 1990s, the process of economic globalization has been accelerated significantly. The globalization of automobile industry, one of the leading and typically global industries, is mainly reflected with two distinct and interrelated characteristics. First is the automobile industry chain, including investment, production, procurement, sales, after-sales service, $R \& D$, and other major links of the increasingly global allocation. For example, in the past, multinational corporations established and maintained research and development institutions in their own countries, investing in target country markets in a replicated way, while now they have developed a way of allocating functional activities and capabilities to global markets. This leads to the emergence of new specialized divisions of labor cooperation mode, especially the separation trend between vehicle assembly and parts manufacturers. More and more multinational companies are involved in manufacturing of parts.
The network organization structure between parts manufacturers and vehicle assembly companies with contracts as the link is becoming increasingly apparent. The global purchase of parts and components in the automobile manufacturing enterprises and the internationalization of the parts industry blur the "national characteristics" of automobile products, which makes automobile products a typical global product. Second is the large-scale reorganization between giant automobile enterprises. Since the 1990s, due to the global excess of automobile production capacity and the increasingly strict regulations on safety, emission, and energy conservation, the pace of global industrial structure adjustment in automobile industry has been accelerated significantly. Many developed countries' auto companies have strengthened their competitiveness by means of expansion, integration, and merger. The trend of automobile industry globalization has a profound impact on the development of automobile industry and industrial policies of such developing countries as China. As a world 
populous country with rising economy, China has become the most potential emerging market in the world with the continuous improvement of people's income level and the upgrading of consumption structure. Improving the consumption environment will become an important measure to transform the potential demand of the public for cars into real demand to promote economic growth. At present, China has initially formed a relatively independent automobile production system. The market advantages, labor quality and cost advantages, and scale advantages of industrial support of large countries are gradually emerging. With the entry of global automobile manufacturing multinational companies and the development of domestic automobile enterprises, China has become an important automobile manufacturing base in the world.

By the end of 2020, China's car ownership has reached 281 million, with 24.24 million newly registered vehicles in 2020, a decrease of 1.53 million, or $5.95 \%$ compared with 2019. Among them, 4.16 million trucks are newly registered, an increase of 650,000 or $18.43 \%$ over 2019 , reaching a new high in the past decade. The number of newly registered motorcycles is 8.26 million, an increase of 2.49 million or $43.07 \%$ over 2019. A rapid growth has been maintained in recent two years, and a significant growth trend would occur in 2020 due to the impact of the epidemic. There are 70 cities in China, each of which has more than 1 million cars, an increase of $4 \%$ over the same period. Each of 31 cities in China has more than 2 million cars. The number exceeds 3 million in 13 cities. There are more than 5 million vehicles each in Beijing, Chengdu, and Chongqing, more than 4 million each in Suzhou, Shanghai, and Zhengzhou and more than 3 million each in seven cities, including Xi'an, Wuhan, Shenzhen, Dongguan, Tianjin, Qingdao, and Shijiazhuang. By the end of 2020, the national new energy vehicle ownership has reached 4.92 million, accounting for $1.75 \%$ of the total automobile, an increase of 1.11 million and $29.18 \%$ over 2019. Among them, the total number of pure electric vehicles is 4 million, accounting for $81.32 \%$ of the total new energy vehicles. The increment of new energy vehicles has exceeded 1 million for three consecutive years, showing a continuous high-speed growth trend.

In 2020, China's traffic management department has handled a total of 25.21 million motor vehicle transfer and registration services. Among them, 24.81 million are transfer business. In the past five years, the proportion of car transfer and registration business volume has increased from 59\% to $102 \%$, reflecting an increasingly active second-hand car trading market. In 2020, the number of motor vehicle drivers in China has reached 456 million. Affected by the novel coronavirus pneumonia, the number of new drivers in China (less than 1 year of driving experience) reached 22,310,000 in 2020 , accounting for $4.90 \%$ of the total number of motorists in the country, a decrease of 7,120,000 and $24.19 \%$ compared with 2019 . From the perspective of driver gender, there are 308 million male drivers, accounting for $67.57 \%$, and 148 million female drivers, accounting for $32.43 \%$. From the perspective of driver age, there are 327 million drivers aged 26 to 50 , accounting for $71.79 \%$; 60.86 million drivers aged 51 to 60 , accounting for $13.36 \%$. Although China's auto market is booming, the luxury car market is mainly monopolized by foreign manufacturers. The analysis of the competitive situation and equilibrium of luxury automobile market is helpful for a better understanding of the operation mechanism of China's automobile market.

The development of any industrial system will be restricted by its own growth ability and resource environment, so the evolution process of any industrial system is limited and regular. Almost all industries will obey the law of cycle and will go through the process from birth to growth to maturity and then to recession. Similarly, the development of the industry should not be unlimited. Due to the constraints of the industry itself and external conditions, there is a problem of limited growth. If an industrial system is regarded as an ecosystem, the enterprises in the industry can be regarded as its population. The enterprise population dynamics model mainly focuses on the change of population quantity, and its law of change is based on the law of nonlinear growth in biological population quantity. Many species in nature grow nonlinearly, and the nonlinear growth of population is also very common. Under the influence of market environment, industrial policy, and development resources in a certain region, the enterprise population may change rapidly.

The competition and coordination mechanism within the population is also an important factor. This setting is based on the principle of intraspecies competition of a biological population. There is competition in the natural biological population, the function of which is to regulate the population size. The larger the population is, the more competition there is. There are also certain competition mechanisms in the innovation population, which will restrain the overexpansion of the innovation population to a certain extent. As a result, intraspecies competition is actually one of the processes for the innovation population to achieve the survival of the fittest. Therefore, this mechanism should also be taken as an important component of the growth model of the entrepreneurial population. There is also competition or synergy among the innovation groups of enterprises in neighboring regions. Lotka-Volterra model is the main method to study the interaction mechanism among populations.

The Lotka-Volterra model was successfully spread to the research fields of social and economic system to interpret the competition behavior between organizations [1]. The model is widely used in the research fields of industrial competition [2], enterprise competition [3], market competition [4], and product competition [5]. Some researchers use the Lotka-Volterra model to analyze innovation activities among competitors [6] and technology substitution among competitors [7]. Some researchers focus on the transformation path [8].

In terms of data characteristics, social economic system is also a grey system. Grey system theory is a method to study and solve a certain system with uncertain information, that is, a grey system. Grey system is one between black system and white system, which contains both known and unknown information. The information contained in a grey system is called grey information. In the abstract systems of society, 
economy, and ecology, researchers should extend the viewpoints and methods of general cybernetics, systems theory, and information theory to those abstract systems and make reasonable explanations. The grey system theory establishes a set of theories and methods to solve the related problems of incomplete information system, which has great development potential in practical application. This theory is a new one, which takes the grey system as the research object and uses a specific method to describe and control the grey system. In fact, the theory is to study the whitening problem of grey system, that is, to study how to use the existing information to predict the unknown information in the future from a systematical perspective. The essence is to use the idea and method of the theory to quantify the abstract phenomenon, analyze the relevant data, and make quantitative prediction and control for the future, so as to complete the system analysis. GM $(1,1)$ model is widely used in the grey system theory.

GM $(1,1)$ model proposed by Deng [9] has been widely applied in many fields [10]. It has been applied as a forecasting approach in various fields, including wafer fabrication [11], opto-electronics industry forecasting [12], electricity forecasting [13], integrated circuit industry forecasting [14], product profit forecasting [15], and vehicle fatality risk forecasting [16]. As a single variable forecasting model, traditional GM $(1,1)$ cannot be used to analyze the long-term relationship between the two variables.

The classical GM $(1,1)$ model cannot be used in bivariate social and economic system. It is necessary to analyze complicated relationships as they influence each other. Therefore, $\mathrm{Wu}$ et al. used the grey Lotka-Volterra model to analyze the relationship between two variables and used the discrete grey Lotka-Volterra model to forecast the values of two variables [17]. Wang et al. applied grey forecast theory with the Lotka-Volterra competition model to explore the dynamic competition between smart TVs and flat panel TVs. After comparing forecast accuracy among the model proposed in this study, they found the proposed model has the best accuracy [5]. Mao et al. [18] established direct grey Lotka-Volterra model with adjustable background value. However, the current application of the grey Lotka-Volterra model mainly discusses the relationship between the two populations and the prediction of population size and does not involve the equilibrium analysis of the Lotka-Volterra model.

In order to make up for the defects of the current research, the research objectives of this paper are as follows: (1) a more accurate grey time series model is constructed for data transformation or prediction; (2) three species grey Lotka-Volterra model is used to analyze the competition of grey time series data; (3) a competitive and equilibrium analysis is made taking three brands of cars in China's luxury car market as an example; and (4) the accuracy of grey time series model and the robustness of the three species grey Lotka-Volterra model are verified based on case data.

The contents and arrangements of the research are as follows: (1) a compound grey transformation system based on GM $(1,1)$ prediction model is constructed to transform the original data; (2) the influence mechanism among enterprise populations is analyzed by the model of three- dimensional grey Lotka-Volterra system; (3) the multichoice goal programming is built to analyze the balanced competition of enterprises. Highlights of this paper are as follows: (1) an improved grey GM $(1,1)$ prediction model is provided; (2) the competition among three enterprises is analyzed based on three-dimensional grey Lotka-Volterra system; and (3) multichoice goal programming approach is introduced in the equilibrium analysis of the grey Lotka-Volterra model.

\section{Method and Material}

This study explores competition relationship among grey Lotka-Volterra system. The methods used in this study are described as follows.

As shown in Figure 1, this paper uses three steps to achieve the research goal. Firstly, a compound grey model is constructed to transform the observation data. Then Lotka-Volterra model is used to calculate the symbiotic relationship of the grey transformed data. Finally, MCGP model is used to analyze population growth, population competition, and competitive equilibrium.

\subsection{Grey Transformation of Data Based on Compound Grey} Model. Grey forecast is an approach based on the GM $(1,1)$ basic model to predict uncertain and incomplete information systems to determine the elements' future dynamic situation among a certain sequence of numbers. The processes of the GM $(1,1)$ model are shown as follows.

The original data series $X^{(0)}$ is

$$
X^{(0)}=\left\{x^{(0)}(1), x^{(0)}(2), \cdots, x^{(0)}(n)\right\} .
$$

The accumulated generating operation (AGO) $X^{(1)}(k)$ is

$$
\left\{\begin{array}{l}
X^{(1)}(k)=\sum_{i=1}^{k} X^{(0)}(i), k=1,2,3, \cdots n \\
X^{(0)}=\left\{x^{(0)}(1), x^{(0)}(2), x^{(0)}(3), \cdots, x^{(0)}(n)\right\}
\end{array}\right.
$$

The original form of GM $(1,1)$ model is

$$
X^{(0)}(k)+a X^{(1)}(k)=b .
$$

The parameter estimation based on the original form of the model and equation (3) is called the original difference grey model (ODGM) [10]. In the GM $(1,1)$ model, the parameters $a$ and $b$ should be calculated first with the method of ordinary least square. The following model is an even GM $(1,1)$ system or even grey model (EGM). Practice shows that the simulation effect of the model is better, and it is also a commonly used grey model.

$$
\left\{\begin{array}{l}
X^{(0)}(k)+a z^{(1)}(k)=b \\
z^{(1)}(k)=\frac{x^{(1)}(k)+x^{(1)}(k+1)}{2} . \\
k=1,2,3, \cdots, n-1
\end{array}\right.
$$




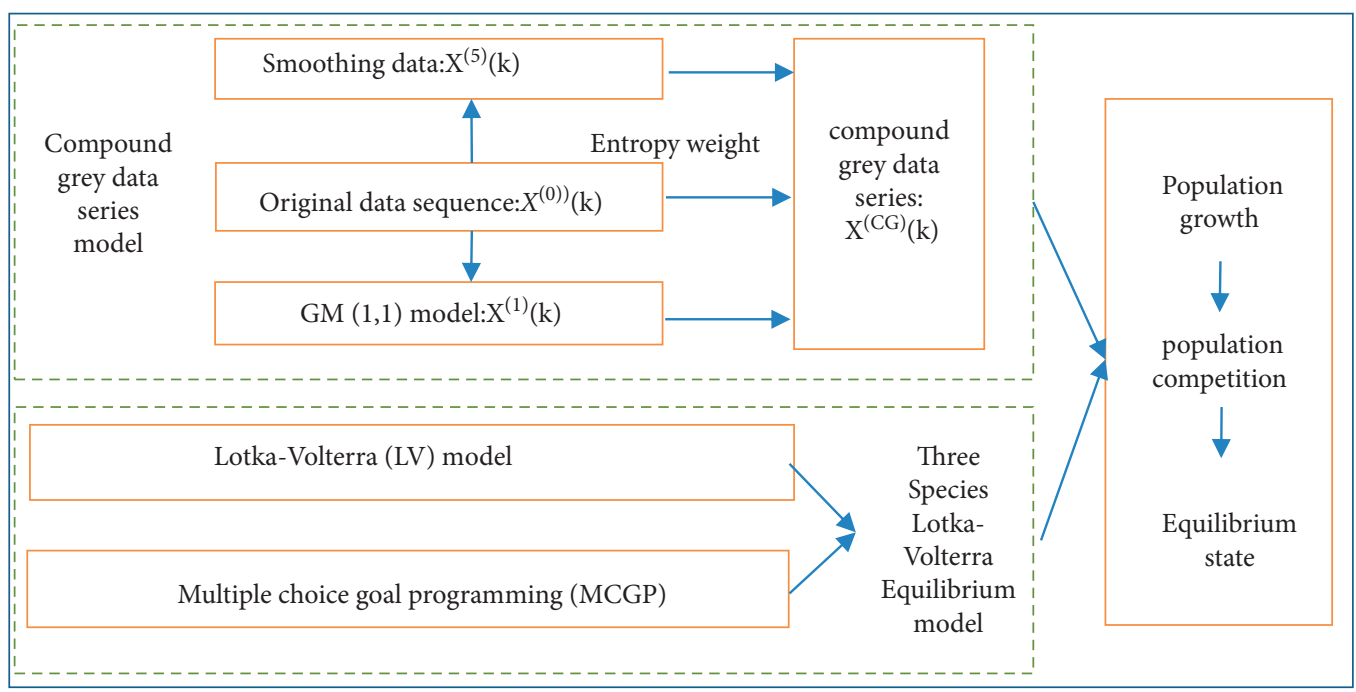

FIGURE 1: Research process.

After estimation of $a$ and $b$, the grey prediction equation can be solved as follows:

$$
\widehat{X}^{(1)}(k+1)=\left[X^{(0)}(1)-\frac{b}{a}\right] e^{-a k}+\frac{b}{a} .
$$

The following can be obtained based on inverse accumulated generating operation (IAGO):

$$
\begin{aligned}
X^{(0)}(k+1) & =X^{(1)}(k+1)-X^{(1)}(k) \\
& =\left[X^{(0)}(1)-\frac{b}{a}\right] e^{-a k}\left(1-e^{a}\right), \quad k=1,2,3, \cdots, n-1 .
\end{aligned}
$$

The new information priority principle is one of the basic principles of the grey system. Many scholars use this principle to optimize the grey GM $(1,1)$ model, but theoretical proof is needed for the priority of new information. Because the priority of the latest information in the traditional grey GM $(1,1)$ prediction model cannot be compared intuitively, this paper constructs a composite GM $(1,1)$ grey data model based on the weight of information entropy in order to make full use of the original data. This paper studies the quarterly sales of three major manufacturers in the luxury car market in mainland China and the grey model is used to transform the observation data in order to obtain more abundant research information. This paper aims at the characteristics of automobile sales data, considering the grey of original data and seasonal characteristics of sales data to construct the compound grey data series $X^{(\mathrm{CG})}(k)$.

$$
X^{(C G)}(k)=w_{1} X^{(0)}(k)+w_{2} X^{(1)}(k)+w_{3} X^{(S)}(k) .
$$

Among them, $X^{(S)}(k)$ is smoothing data series for seasons, and $w_{i}$ is entropy weight. Entropy weight is an objective method [19]. It is a popular method and is always combined with other methods. For example, entropy combined with fuzzy VIKOR [20], with GIS and AHP [21], or with subjective weight [22]. Entropy weight method is provided as follows [23]. Supposing there are $m$ alternatives $\left(A_{1}, A_{2}, \ldots, A_{m}\right)$ and $n$ criteria $\left(C_{1}, C_{2}, \ldots, C_{n}\right)$ for a decision problem, then the initial decision matrix is

$$
A=\left[\begin{array}{cccc}
a_{11} & a_{12} & \cdots & a_{1 n} \\
a_{21} & a_{22} & \cdots & a_{2 n} \\
\vdots & \vdots & \ddots & \vdots \\
a_{m 1} & a_{m 2} & \cdots & a_{m n}
\end{array}\right]=\left[a_{i j}\right]_{m \times n}
$$

Step 1 : normalize the evaluation matrix:

$$
r_{i j}=\frac{a_{i j}}{\sqrt{\sum_{i=1}^{m} a_{i j}^{2}}} .
$$

Step 2 : compute entropy:

$$
e_{j}=-\frac{1}{\ln m} \sum_{i=1}^{m} r_{i j} \ln r_{i j}, \quad j=1,2, \cdots, n .
$$

Step 3 : calculate weights:

$$
w_{j}=\frac{1-e_{j}}{\sum_{i=1}^{n}\left(1-e_{j}\right)}, \quad j=1,2, \cdots, n
$$

In order to further test the prediction accuracy of the compound grey model, this paper compares the prediction results of grey prediction model and autoregression moving average (ARMA) model that is commonly used in time series analysis.

2.2. Competitive Situation and Equilibrium Analysis Based on the Lotka-Volterra Model. The paper constructs an internal dynamic system of population $1\left(P_{1}\right)$ based on the logistic model. 


$$
g_{1}(t)=\frac{d N_{1}(t)}{d t}=\alpha_{1} N_{1}\left(1-\frac{N_{1}}{K_{1}}\right)
$$

where $g_{1}(t)$ is the growth rate in phase $t$ and $N_{1}(t)$ is population size of period $t . K_{1}$ is the maximum population scale; $\alpha_{1}$ is the intrinsic growth rate, and $\left(1-N_{1} / K_{1}\right)$ is the retardation of growth.

The measurement model is as follows.

Because $\quad d N_{1}(t) \approx \Delta N_{1}(t), \quad \Delta N_{1}(t)=N_{1}(t)-N_{1}$ $(t-1), d t \approx \Delta t=t-(t-1)=1$.

So,

$$
g_{1}(t) \approx \Delta N(t)=\gamma_{1} N_{1}(t-1)+\gamma_{2} N_{1}^{2}(t-1) .
$$

Among them, $\gamma_{1}=\alpha_{1}$, usually $\gamma_{1}>1$. It usually represents the synergy within a population. $\gamma_{2}=-\alpha_{1} / K_{1}$, usually, $\gamma_{2}<0$. It refers to the competition effect within a population. It is called the internal competition coefficient or population density inhibition coefficient.

Similarly, the internal relation model of population 2 $\left(P_{2}\right)$ can be obtained:

$$
g_{2}(t)=\frac{d N_{2}(t)}{d t}=\alpha_{2} N_{2}\left(1-\frac{N_{2}}{K_{2}}\right) .
$$

The following system shows the impact of $P_{2}$ on $P_{1}$ :

$$
g_{1}(t)=\frac{d N_{1}(t)}{d t}=\alpha_{1} N_{1}\left(1-\frac{N_{1}}{K_{1}}+\frac{\beta_{12} N_{2}}{K_{2}}\right),
$$

where $\beta_{12}$ is the influence of population 2 on population 1 $\left(\beta_{12}>0\right.$, synergistic effect; $\beta_{12}<0$, competitive effect). Similarly, the following system shows the impact of $\mathrm{P}_{1}$ on $\mathrm{P}_{2}$ :

$$
g_{2}(t)=\frac{d N_{2}(t)}{d t}=\alpha_{2} N_{2}\left(1-\frac{N_{2}}{K_{2}}+\frac{\beta_{21} N_{1}}{K_{1}}\right),
$$

where $\beta_{21}$ is the impact factor of population 1 on population 2. The dynamic system of $P_{1}$ and $P_{2}$ is

$$
\left\{\begin{array}{l}
g_{1}(t)=\frac{d N_{1}(t)}{d t}=\alpha_{1} N_{1}\left(1-\frac{N_{1}}{K_{1}}+\frac{\beta_{12} N_{2}}{K_{2}}\right) \\
g_{2}(t)=\frac{d N_{2}(t)}{d t}=\alpha_{2} N_{2}\left(1-\frac{N_{2}}{K_{2}}+\frac{\beta_{21} N_{1}}{K_{1}}\right)
\end{array} .\right.
$$

Equation (17) is called the Lotka-Volterra (LV) system, which is based on the logistic model of a single species, and considers the dynamic growth of competition and symbiosis of two or more entities simultaneously in the ecosystem [24]. LV model can not only accurately describe the competition and symbiosis between corporate populations, but also determine the influence of the core population in the evolution of the entire ecosystem [25]. The LV system has better data fitting and prediction expression [26].

The classical LV model is used to simulate the dynamic relationship of populations in an ecological system. It was introduced into the fluctuation of macro-economic growth and the market competition of medium scale and scope. According to the principle of biology, there are two kinds of functional relationships among biological populations: promotion or inhibition. For their own survival and development, there is also such a relationship between market competition subjects: the existence of one subject can promote or inhibit the diffusion process of another subject. Based on the numerical value of $\beta_{\mathrm{ij}}$, the type of interaction between species can be judged [27]. In a symbiotic system composed of population $1\left(P_{1}\right)$, population $2\left(P_{2}\right)$, and population $3\left(P_{3}\right)$, the $\mathrm{LV}$ model is

$$
\left\{\begin{array}{l}
g_{1}(t)=\frac{d N_{1}(t)}{d t}=\alpha_{1} N_{1}\left(1-\frac{N_{1}}{K_{1}}+\frac{\beta_{12} N_{2}}{K_{2}}+\frac{\beta_{13} N_{3}}{K_{3}}\right) \\
g_{2}(t)=\frac{d N_{2}(t)}{d t}=\alpha_{2} N_{2}\left(1-\frac{N_{2}}{K_{2}}+\frac{\beta_{21} N_{1}}{K_{1}}+\frac{\beta_{23} N_{3}}{K_{3}}\right) \\
g_{3}(t)=\frac{d N_{3}(t)}{d t}=\alpha_{3} N_{3}\left(1-\frac{N_{3}}{K_{3}}+\frac{\beta_{31} N_{1}}{K_{1}}+\frac{\beta_{32} N_{2}}{K_{2}}\right)
\end{array} .\right.
$$

Among them, $\beta_{i j}(i=1,2,3 \cdot j=1,2,3$. $)$ is the interaction coefficient between populations. When $\beta_{i j}$ is greater than zero, it indicates that there is a synergetic relationship among populations. When $\beta_{i j}$ is less than zero, it means that there is competition among populations.

The following is econometric model (19) and regression analysis:

$$
\left\{\begin{array}{l}
N_{1}(t)=\gamma_{11} N_{1}(t-1)+\gamma_{12} N_{1}^{2}(t-1)+\gamma_{13} N_{1}(t-1) N_{2}(t-1)+\gamma_{14} N_{1}(t-1) N_{3}(t-1) \\
N_{2}(t)=\gamma_{21} N_{2}(t-1)+\gamma_{22} N_{2}^{2}(t-1)+\gamma_{23} N_{2}(t-1) N_{1}(t-1)+\gamma_{24} N_{2}(t-1) N_{3}(t-1) \\
N_{3}(t)=\gamma_{31} N_{3}(t-1)+\gamma_{32} N_{3}^{2}(t-1)+\gamma_{33} N_{3}(t-1) N_{1}(t-1)+\gamma_{34} N_{3}(t-1) N_{2}(t-1)
\end{array} .\right.
$$

Among them, $\gamma_{11}=\alpha_{1}+1, \gamma_{12}=-\alpha_{1} / K_{1}, \gamma_{13}=\alpha_{1} \beta_{12} /$ $K_{2}$, and $\gamma_{14}=\alpha_{1} \beta_{13} / K_{3}$. Generally, $\gamma_{11}>1, \alpha_{1}>0$, it means the synergy effect within the population 1 , and $\gamma_{12}<0$, it means the competition effect within the population. It is called the internal competition coefficient or the population density inhibition coefficient. In the same way: $\gamma_{21}=\alpha_{2}+1$, $\gamma_{22}=-\alpha_{2} / K_{2}, \gamma_{23}=\alpha_{2} \beta_{21} / K_{1}, \gamma_{24}=\alpha_{2} \beta_{23} / K_{3}, \gamma_{31}=\alpha_{3}+1$, $\gamma_{32}=-\alpha_{3} / K_{3}, \gamma_{33}=\alpha_{3} \beta_{31} / K_{1}$, and $\gamma_{34}=\alpha_{3} \beta_{32} / K_{2}$.

2.3. Population Growth under Constraints of Three Species Lotka-Volterra Equilibrium. Objective programming is an 
effective way to solve the problem of multiobjective programming. The basic idea is to determine the expected value of each objective function of the multiobjective programming problem. The goal programming method is more flexible, effective, and easy to use and implement in dealing with multiobjective problems. In recent years, multichoice goal programming (MCGP) has been widely used to solve many practical decision problems [28]. Chang [29] proposed the multiple choice goal programming (MCGP) method as

$$
\left\{\begin{array}{l}
\text { Objective function, } \\
\operatorname{Min} \sum_{i=1}^{n}\left(d_{i}^{+}+d_{i}^{-}\right)+\sum_{i=1}^{n}\left(e_{i}^{+}+e_{i}^{-}\right) \\
\text {Constraints, } \\
g_{i}=f_{i}(x)-d_{i}^{+}+d_{i}^{-}, \quad i=1,2, \cdots, n \\
x \in X=\left\{x_{1}, x_{2}, \cdots, x_{m}\right\} \\
X \in F, \quad(F \text { is the set of feasible solutions }) \\
g_{i, \max }=g_{i}-e_{i}^{+}+e_{i}^{-}, \quad i=1,2, \cdots, n \\
g_{i, \max } \geq g_{i} \geq g_{i, \min }, \quad i=1,2, \cdots, n \\
e_{i}^{+}, e_{i}^{-}, d_{i}^{+}, d_{i}^{-} \geq 0, \quad i=1,2, \cdots, n
\end{array}\right.
$$

Here, $d_{i}^{+}, d_{i}^{-}$indicate the value of the $i$-th goal exceeding and not reaching the expected value of the goal. $f_{i}(x)$ is the formula of the $i$-th objective. $X$ is the decision variable. $g_{i}$ indicates the expected goal of $i$-th. $e_{i}^{+}$are close to positive deviation of $\left|g_{i}-g_{i, \max }\right| \cdot e_{i}^{-}$are close to negative deviation of $\left|g_{i}-g_{i, \max }\right| \cdot g_{i, \min }$ and $g_{i, \max }$ are the lower and upper limits for the goal of $g_{i}$. Having learned from the relevant research $[30,31]$, the paper has embedded the equilibrium condition of LV system into the MCGP model to obtain the Lotka-Volterra-MCGP model:

Objective function, $\operatorname{Min} \sum_{i=1}^{n}\left(d_{i}^{+}+d_{i}^{-}\right)+\sum_{i=1}^{n}\left(e_{i}^{+}+e_{i}^{-}\right)$

$$
\left\{\begin{array}{l}
\text { Constraints } \\
g_{i}=f_{i}(x)+d_{i}^{-}-d_{i}^{+}, \quad i=1,2, \cdots, n \\
x \in X, X=\left\{x_{1}, x_{2}, \cdots, x_{m}\right\} \\
X \in F \quad(F \text { is the set of feasible solutions }) \\
g_{i, \text { max }}=g_{i}+e_{i}^{-}-e_{i}^{+}, \quad i=1,2, \cdots, n \\
g_{i, \text { min }} \leq g_{i}, g_{i} \leq g_{i, \text { max }}, \quad i=1,2, \cdots, n \\
d_{i}^{+}, d_{i}^{-}, e_{i}^{+}, e_{i}^{-} \geq 0, \quad i=1,2, \cdots, n \\
g_{1}(t)=\frac{d N_{1}(t)}{d t}=\alpha_{1} N_{1}\left(1-\frac{N_{1}}{K_{1}}+\frac{\beta_{12} N_{2}}{K_{2}}+\frac{\beta_{13} N_{3}}{K_{3}}\right) \\
g_{2}(t)=\frac{d N_{2}(t)}{d t}=\alpha_{2} N_{2}\left(1-\frac{N_{2}}{K_{2}}+\frac{\beta_{21} N_{1}}{K_{1}}+\frac{\beta_{23} N_{3}}{K_{3}}\right) \\
g_{3}(t)=\frac{d N_{3}(t)}{d t}=\alpha_{3} N_{3}\left(1-\frac{N_{3}}{K_{3}}+\frac{\beta_{31} N_{1}}{K_{1}}+\frac{\beta_{32} N_{2}}{K_{2}}\right) \\
-1<\beta_{i j}<1, i=1,2,3 . j=1,2,3 . \\
\sum g_{i}=K,(K \text { is the sumof market capacity })
\end{array}\right.
$$


As a linear form for objective programming, multichoice goal programming embedded with Lotka-Volterra equilibrium (LV-MCGP) can be easily resolved by any common software.

2.4. Empirical Analysis. The sales volume of luxury brand cars in mainland China has achieved strong growth again in 2020. According to the domestic automobile wholesale data provided by China passenger car federation, the sales volume of luxury car market in 2020 has been 2.79 million, with a year-on-year increase of $19.9 \%$. The sales volume of China's automobile market has declined in the three consecutive years from 2018 to 2020 by $3.9 \%, 9.2 \%$, and $6.3 \%$ respectively. However, sales of luxury cars have risen by $17.6 \%, 11.7 \%$, and $19.9 \%$, respectively, in the same period. In the passenger car federation's wholesale list, Audi won the sales champion. Audi, Mercedes Benz, and BMW sell $638,000,619,000$, and 609,000 domestic models, respectively, in 2020. ABB (representing Audi, Benz, and BMW) has contributed 1.866 million in total, accounting for $67 \%$ of the total luxury car market. The competition among the three luxury brands in first group is increasingly fierce. This situation will continue in 2021 and in the next three years, and the competition will be more intense. In 2020, China's luxury car consumption market has achieved strong growth and has become the focus of global car market. In 2021, global automobile luxury brand manufacturers will pay more attention to and rely more on the Chinese market. With the positive progress of vaccine delivery, the global economy is expected to enter the repair period in 2021, and the uncertainty of China's economic development will be greatly reduced compared with 2020. In 2021, China's luxury car market is expected to maintain a strong growth. This paper will analyze the market competition of the three main luxury brands based on the data obtained by the composite grey transformation.

Two years ago, BMW completed the change of its share ratio in the BMW Brilliance joint venture. The $75 \%$ shareholding means that BMW will get a higher profit than Mercedes Benz and Audi whenever it sells a BMW, so it will be in a more comfortable position in the competition. The high $75 \%$ shareholding ratio also means that BMW's investment in the Chinese market is guaranteed and the speed of model introduction is accelerated. Since 2021, BMW's production capacity in mainland China will rapidly rise to the level of an annual output of 800,000 vehicles after the expansion of BMW Brilliance's third factory. In addition, BMW's i-series new products can basically achieve synchronous sales with the international market since the news of pure electric vehicles.

As shown in Table 1, the process of grey transformation is easy to realize. Entropy weight is calculated based on the data in the example: $w_{1}=0.333, w_{2}=0.333$, and $w_{3}=0.333$. It shows that there is no difference between the original data, seasonal smoothing data, and GM $(1,1)$ data in the evaluation of information entropy. It also shows that the information fidelity of grey transformation is relatively high.
Mercedes Benz will launch a new generation of S-class in 2021. The S-class is always the leader of the new design language, which means that Mercedes Benz will start the wave of product upgrading again from 2021. In 2021, the C-class car will also be upgraded, which is obviously faster than in previous years.

As shown in Table 2, the Benz sales data of grey transformation is easy to realize. Similar to BMW's data, $w_{1}=0.333, w_{2}=0.333$, and $w_{3}=0.333$. It shows that there is no difference between the original data, seasonal smoothing data, and GM $(1,1)$ data in the evaluation of information entropy. It also shows that the information fidelity of grey transformation is relatively high.

Audi is trying its best to consolidate its leading position in China's luxury car market. By the end of 2020, SAIC Audi has officially announced that Audi will become the first luxury brand with two major vehicle production and sales joint ventures in China, which means that Audi will speed up again in the sales campaign of luxury brand vehicles. In 2021, SAIC Audi will release its first domestic Audi a7l, which is expected to be officially launched in 2022, and is likely to speed up the process. Audi and China First Automobile Group's new energy vehicle joint venture company in Jilin Province also signed a contract in January 2021. The first phase plant plans to invest 30 billion yuan, while Audi and Volkswagen group hold $60 \%$ of the equity of the new company. New investment and new share ratio help Audi lock in its competitive advantage in the next 10 years.

As shown in Table 3, the Audi sales data of grey transformation is realized. In this paper, the mean absolute percentage error (MAPE) index is used to evaluate the effect of grey transformation, Among them,

$$
\text { MAPE }=\frac{1}{n} \sum_{k=1}^{n}\left|\frac{A_{k}-F_{k}}{A_{k}}\right| \times 100 \%,
$$

where $A_{k}$ is the actual value and $F_{k}$ is the predicted value.

As shown in Table 4, the prediction effect of composite prediction model is very good. The ARMA model has the greatest error. The compound grey model has the smallest error. The practice shows that among the four prediction models, the compound grey model has the best prediction accuracy.

As shown in Figure 2, the radar chart shapes of different prediction models of the three enterprises are similar, which shows the stability of the prediction accuracy of the model. The ARMA model has the worst prediction accuracy. The compound grey system has more variety and more practical advantages. For example, (1) the compound grey model can be used to explain population dynamics. Compound grey model is an improvement of traditional population dynamics model, which is suitable for the research of ecology related fields. (2) It can be constructed as a generalized prediction model with time as the independent variable. (3) The application scenarios are more extensive than ARMA model, especially in the case of insufficient or missing original data. 
TABLE 1: Grey transformation data of BMW sales volume.

\begin{tabular}{|c|c|c|c|c|c|c|c|c|c|c|c|}
\hline Year-month & $X^{(0)}(k)$ & $X^{(S)}(k)$ & $X^{(1)}(k)$ & $X^{(\mathrm{CG})}(k)$ & ARMA & Year-month & $X^{(0)}(k)$ & $X^{(\mathrm{S})}(k)$ & $X^{(1)}(k)$ & $X^{(\mathrm{CG})}(k)$ & ARMA \\
\hline $2016-1$ & 27663 & & & & & & & & & & \\
\hline $2016-2$ & 13103 & & 24175 & & 27077 & $2018-8$ & 36698 & 34054 & 37913 & 36222 & 33225 \\
\hline 2016-3 & 24324 & 21697 & 24541 & 23520 & 12538 & $2018-9$ & 37638 & 36052 & 38486 & 37392 & 36098 \\
\hline 2016-4 & 21736 & 19721 & 24912 & 22123 & 23743 & 2018-10 & 46925 & 40420 & 39067 & 42138 & 37037 \\
\hline $2016-5$ & 24192 & 23417 & 25288 & 24299 & 21159 & 2018-11 & 51460 & 45341 & 39658 & 45486 & 46310 \\
\hline $2016-6$ & 25693 & 23874 & 25670 & 25079 & 23611 & 2018-12 & 41815 & 46733 & 40257 & 42935 & 50839 \\
\hline 2016-7 & 24802 & 24896 & 26058 & 25252 & 25110 & 2019-1 & 53289 & 48855 & 40865 & 47670 & 41208 \\
\hline $2016-8$ & 28124 & 26206 & 26452 & 26927 & 24220 & 2019-2 & 32929 & 42678 & 41483 & 39030 & 52665 \\
\hline $2016-9$ & 30709 & 27878 & 26852 & 28480 & 27537 & 2019-3 & 49482 & 45233 & 42110 & 45608 & 32335 \\
\hline 2016-10 & 25276 & 28036 & 27257 & 26857 & 30118 & 2019-4 & 49945 & 44119 & 42746 & 45603 & 48863 \\
\hline 2016-11 & 31125 & 29037 & 27669 & 29277 & 24693 & 2019-5 & 46602 & 48676 & 43392 & 46223 & 49326 \\
\hline 2016-12 & 26461 & 27621 & 28087 & 27390 & 30534 & $2019-6$ & 42315 & 46287 & 44048 & 44217 & 45988 \\
\hline $2017-1$ & 31586 & 29724 & 28512 & 29941 & 25877 & 2019-7 & 45710 & 44876 & 44713 & 45100 & 41707 \\
\hline $2017-2$ & 26287 & 28111 & 28943 & 27780 & 30994 & $2019-8$ & 47644 & 45223 & 45389 & 46085 & 45097 \\
\hline $2017-3$ & 32279 & 30051 & 29380 & 30570 & 25703 & 2019-9 & 49631 & 47662 & 46075 & 47789 & 47028 \\
\hline 2017-4 & 34835 & 31134 & 29824 & 31931 & 31686 & 2019-10 & 48862 & 48712 & 46771 & 48115 & 49012 \\
\hline $2017-5$ & 31505 & 32873 & 30275 & 31551 & 34238 & 2019-11 & 53951 & 50815 & 47478 & 50748 & 48244 \\
\hline $2017-6$ & 27566 & 31302 & 30732 & 29867 & 30913 & 2019-12 & 52577 & 51797 & 48195 & 50856 & 53326 \\
\hline $2017-7$ & 30050 & 29707 & 31197 & 30318 & 26980 & 2020-1 & 48432 & 51653 & 48924 & 49670 & 51954 \\
\hline $2017-8$ & 32888 & 30168 & 31668 & 31575 & 29460 & $2020-2$ & 8039 & 36349 & 49663 & 31350 & 47815 \\
\hline $2017-9$ & 34496 & 32478 & 32147 & 33040 & 32294 & $2020-3$ & 43263 & 33245 & 50414 & 42307 & 7482 \\
\hline 2017-10 & 30420 & 32601 & 32632 & 31885 & 33900 & $2020-4$ & 56204 & 35835 & 51175 & 47738 & 42654 \\
\hline 2017-11 & 38097 & 34338 & 33126 & 35187 & 29830 & $2020-5$ & 57903 & 52457 & 51949 & 54103 & 55576 \\
\hline $2017-12$ & 35548 & 34688 & 33626 & 34621 & 37495 & $2020-6$ & 46597 & 53568 & 52734 & 50966 & 57272 \\
\hline 2018-1 & 43524 & 39056 & 34134 & 38905 & 34950 & $2020-7$ & 63596 & 56032 & 53531 & 57720 & 45983 \\
\hline 2018-2 & 24968 & 34680 & 34650 & 31433 & 42914 & $2020-8$ & 65558 & 58584 & 54340 & 59494 & 62957 \\
\hline 2018-3 & 35569 & 34687 & 35174 & 35143 & 24386 & $2020-9$ & 56350 & 61835 & 55161 & 57782 & 64916 \\
\hline 2018-4 & 36233 & 32257 & 35705 & 34732 & 34971 & $2020-10$ & 47166 & 56358 & 55994 & 53173 & 55721 \\
\hline 2018-5 & 35516 & 35773 & 36245 & 35844 & 35634 & $2020-11$ & 61219 & 54912 & 56840 & 57657 & 46551 \\
\hline 2018-6 & 31644 & 34464 & 36792 & 34300 & 34918 & $2020-12$ & 54834 & 54406 & 57699 & 55647 & 60583 \\
\hline 2018-7 & 33820 & 33660 & 37348 & 34943 & 31052 & 2021-1 & 73333 & 63129 & 58571 & 65011 & 54208 \\
\hline
\end{tabular}

TABLE 2: Grey transformation data of Mercedes Benz sales.

\begin{tabular}{|c|c|c|c|c|c|c|c|c|c|c|c|}
\hline Year-month & $X^{(0)}(k)$ & $X^{(S)}(k)$ & $X^{(1)}(k)$ & $X^{(\mathrm{CG})}(k)$ & ARMA & Year-month & $X^{(0)}(k)$ & $X^{(S)}(k)$ & $\mathrm{X}^{(1)}(k)$ & $X^{(\mathrm{CG})}(k)$ & ARMA \\
\hline 2016-1 & 29256 & & & & & & & & & & \\
\hline $2016-2$ & 17716 & & 27301 & & 28759 & $2018-8$ & 40005 & 41685 & 40322 & 40671 & 40900 \\
\hline $2016-3$ & 23837 & 23603 & 27659 & 25033 & 17240 & 2018-9 & 43970 & 41798 & 40849 & 42206 & 39488 \\
\hline $2016-4$ & 23304 & 21619 & 28020 & 24314 & 23350 & $2018-10$ & 31922 & 38632 & 41384 & 37313 & 43445 \\
\hline $2016-5$ & 25970 & 24370 & 28387 & 26242 & 22818 & 2018-11 & 15419 & 30437 & 41925 & 29260 & 31420 \\
\hline $2016-6$ & 27693 & 25656 & 28758 & 27369 & 25479 & 2018-12 & 49530 & 32290 & 42474 & 41431 & 14948 \\
\hline $2016-7$ & 25009 & 26224 & 29135 & 26789 & 27199 & 2019-1 & 56231 & 40393 & 43030 & 46551 & 48995 \\
\hline $2016-8$ & 25205 & 25969 & 29516 & 26897 & 24520 & 2019-2 & 39595 & 48452 & 43593 & 43880 & 55683 \\
\hline $2016-9$ & 24333 & 24849 & 29902 & 26361 & 24715 & 2019-3 & 55157 & 50328 & 44163 & 49883 & 39078 \\
\hline $2016-10$ & 24279 & 24606 & 30293 & 26393 & 23845 & $2019-4$ & 46252 & 47001 & 44741 & 45998 & 54611 \\
\hline $2016-11$ & 29394 & 26002 & 30690 & 28695 & 23791 & $2019-5$ & 49952 & 50454 & 45326 & 48577 & 45723 \\
\hline 2016-12 & 34582 & 29418 & 31091 & 31697 & 28896 & $2019-6$ & 47544 & 47916 & 45919 & 47126 & 49416 \\
\hline $2017-1$ & 42077 & 35351 & 31498 & 36309 & 34075 & 2019-7 & 49561 & 49019 & 46520 & 48367 & 47013 \\
\hline $2017-2$ & 33862 & 36840 & 31910 & 34204 & 41556 & $2019-8$ & 50366 & 49157 & 47129 & 48884 & 49026 \\
\hline $2017-3$ & 31042 & 35660 & 32327 & 33010 & 33356 & 2019-9 & 51327 & 50418 & 47745 & 49830 & 49829 \\
\hline $2017-4$ & 34613 & 33172 & 32750 & 33512 & 30541 & 2019-10 & 47790 & 49828 & 48370 & 48662 & 50788 \\
\hline $2017-5$ & 37440 & 34365 & 33179 & 34995 & 34106 & 2019-11 & 49653 & 49590 & 49003 & 49415 & 47258 \\
\hline $2017-6$ & 34555 & 35536 & 33613 & 34568 & 36927 & 2019-12 & 50396 & 49280 & 49644 & 49773 & 49118 \\
\hline $2017-7$ & 37205 & 36400 & 34053 & 35886 & 34048 & 2020-1 & 44411 & 48153 & 50293 & 47619 & 49859 \\
\hline $2017-8$ & 35521 & 35760 & 34498 & 35260 & 36693 & $2020-2$ & 11295 & 35367 & 50951 & 32538 & 43885 \\
\hline $2017-9$ & 37495 & 36740 & 34950 & 36395 & 35012 & $2020-3$ & 48589 & 34765 & 51618 & 44991 & 10831 \\
\hline $2017-10$ & 34484 & 35833 & 35407 & 35241 & 36982 & $2020-4$ & 52806 & 37563 & 52293 & 47554 & 48056 \\
\hline 2017-11 & 36936 & 36305 & 35870 & 36370 & 33977 & $2020-5$ & 58431 & 53275 & 52977 & 54895 & 52265 \\
\hline 2017-12 & 41518 & 37646 & 36340 & 38501 & 36424 & $2020-6$ & 52510 & 54582 & 53671 & 53588 & 57879 \\
\hline
\end{tabular}


TABle 2: Continued.

\begin{tabular}{|c|c|c|c|c|c|c|c|c|c|c|c|}
\hline Year-month & $X^{(0)}(k)$ & $X^{(\mathrm{S})}(k)$ & $X^{(1)}(k)$ & $X^{(\mathrm{CG})}(k)$ & ARMA & Year-month & $X^{(0)}(k)$ & $X^{(\mathrm{S})}(k)$ & $\mathrm{X}^{(1)}(k)$ & $X^{(\mathrm{CG})}(k)$ & ARMA \\
\hline 2018-1 & 56306 & 44920 & 36815 & 46014 & 40998 & $2020-7$ & 55720 & 55554 & 54373 & 55215 & 51969 \\
\hline $2018-2$ & 33564 & 43796 & 37297 & 38219 & 55758 & $2020-8$ & 55241 & 54490 & 55084 & 54939 & 55173 \\
\hline $2018-3$ & 43051 & 44307 & 37785 & 41714 & 33059 & $2020-9$ & 63008 & 57990 & 55805 & 58934 & 54695 \\
\hline 2018-4 & 44006 & 40207 & 38279 & 40831 & 42528 & $2020-10$ & 53964 & 57404 & 56535 & 55968 & 62448 \\
\hline 2018-5 & 44589 & 43882 & 38780 & 42417 & 43481 & 2020-11 & 62451 & 59808 & 57275 & 59844 & 53421 \\
\hline $2018-6$ & 43631 & 44075 & 39287 & 42331 & 44063 & 2020-12 & 60624 & 59013 & 58024 & 59220 & 61892 \\
\hline 2018-7 & 41420 & 43213 & 39801 & 41478 & 43107 & 2021-1 & 68412 & 63829 & 58783 & 63675 & 60068 \\
\hline
\end{tabular}

TABLE 3: Grey transformation data of Audi car sales.

\begin{tabular}{|c|c|c|c|c|c|c|c|c|c|c|c|}
\hline Year-month & $X^{(0)}(k)$ & $X^{(S)}(k)$ & $X^{(1)}(k)$ & $X^{(\mathrm{CG})}(k)$ & ARMA & Year-month & $X^{(0)}(k)$ & $X^{(\mathrm{S})}(k)$ & $X^{(1)}(k)$ & $X^{(\mathrm{CG})}(k)$ & ARMA \\
\hline $2016-1$ & 55285 & & & & & & & & & & \\
\hline $2016-2$ & 32994 & & 41006 & & 54906 & $2018-8$ & 55781 & 45431 & 49093 & 50102 & 38383 \\
\hline $2016-3$ & 49537 & 45939 & 41253 & 45576 & 32627 & $2018-9$ & 61015 & 51850 & 49388 & 54084 & 55401 \\
\hline $2016-4$ & 47440 & 43324 & 41501 & 44088 & 49161 & $2018-10$ & 46988 & 54595 & 49685 & 50423 & 60632 \\
\hline $2016-5$ & 46644 & 47874 & 41751 & 45423 & 47065 & 2018-11 & 51842 & 53282 & 49984 & 51703 & 46613 \\
\hline $2016-6$ & 44011 & 46032 & 42002 & 44015 & 46269 & 2018-12 & 52881 & 50570 & 50285 & 51245 & 51465 \\
\hline $2016-7$ & 43746 & 44800 & 42255 & 43600 & 43638 & 2019-1 & 50987 & 51903 & 50588 & 51159 & 52503 \\
\hline $2016-8$ & 45512 & 44423 & 42509 & 44148 & 43373 & $2019-2$ & 30295 & 44721 & 50892 & 41969 & 50610 \\
\hline $2016-9$ & 46075 & 45111 & 42765 & 44650 & 45138 & $2019-3$ & 48821 & 43368 & 51198 & 47796 & 29930 \\
\hline $2016-10$ & 43193 & 44927 & 43022 & 43714 & 45701 & $2019-4$ & 40334 & 39817 & 51506 & 43886 & 48445 \\
\hline 2016-11 & 54789 & 48019 & 43281 & 48696 & 42820 & $2019-5$ & 37320 & 42158 & 51816 & 43765 & 39963 \\
\hline 2016-12 & 27063 & 41682 & 43542 & 37429 & 54410 & $2019-6$ & 50666 & 42773 & 52128 & 48522 & 36951 \\
\hline 2017-1 & 51069 & 44307 & 43804 & 46393 & 26699 & $2019-7$ & 53808 & 47265 & 52442 & 51171 & 50289 \\
\hline $2017-2$ & 28694 & 35609 & 44067 & 36123 & 50692 & $2019-8$ & 55166 & 53213 & 52757 & 53712 & 53429 \\
\hline $2017-3$ & 41506 & 40423 & 44333 & 42087 & 28329 & 2019-9 & 62283 & 57086 & 53075 & 57481 & 54787 \\
\hline $2017-4$ & 42444 & 37548 & 44599 & 41530 & 41134 & 2019-10 & 63301 & 60250 & 53394 & 58982 & 61900 \\
\hline 2017-5 & 44406 & 42785 & 44868 & 44020 & 42072 & 2019-11 & 65684 & 63756 & 53715 & 61052 & 62917 \\
\hline $2017-6$ & 43717 & 43522 & 45138 & 44126 & 44033 & 2019-12 & 60546 & 63177 & 54039 & 59254 & 65299 \\
\hline $2017-7$ & 48232 & 45452 & 45409 & 46364 & 43344 & 2020-1 & 53888 & 60039 & 54364 & 56097 & 60164 \\
\hline $2017-8$ & 52377 & 48109 & 45683 & 48723 & 47857 & $2020-2$ & 8105 & 40846 & 54691 & 34547 & 53509 \\
\hline $2017-9$ & 59382 & 53330 & 45957 & 52890 & 51999 & 2020-3 & 34753 & 32249 & 55020 & 40674 & 7752 \\
\hline $2017-10$ & 51994 & 54584 & 46234 & 50937 & 59000 & $2020-4$ & 55626 & 32828 & 55351 & 47935 & 34385 \\
\hline 2017-11 & 57294 & 56223 & 46512 & 53343 & 51616 & $2020-5$ & 56473 & 48951 & 55684 & 53703 & 55246 \\
\hline 2017-12 & 30889 & 46726 & 46792 & 41469 & 56914 & $2020-6$ & 56588 & 56229 & 56019 & 56279 & 56093 \\
\hline 2018-1 & 62741 & 50308 & 47074 & 53374 & 30523 & $2020-7$ & 58893 & 57318 & 56356 & 57522 & 56208 \\
\hline $2018-2$ & 32883 & 42171 & 47357 & 40804 & 62357 & 2020-8 & 60629 & 58703 & 56695 & 58676 & 58512 \\
\hline $2018-3$ & 53557 & 49727 & 47642 & 50309 & 32516 & $2020-9$ & 72298 & 63940 & 57037 & 64425 & 60247 \\
\hline $2018-4$ & 46422 & 44287 & 47929 & 46213 & 53179 & $2020-10$ & 63998 & 65642 & 57380 & 62340 & 71909 \\
\hline $2018-5$ & 45402 & 48460 & 48217 & 47360 & 46048 & 2020-11 & 66756 & 67684 & 57725 & 64055 & 63614 \\
\hline $2018-6$ & 41759 & 44528 & 48507 & 44931 & 45028 & $2020-12$ & 50301 & 60352 & 58072 & 56242 & 66370 \\
\hline $2018-7$ & 38753 & 41971 & 48799 & 43174 & 41387 & 2021-1 & 80008 & 65688 & 58422 & 68039 & 49924 \\
\hline
\end{tabular}

As shown in Table 5, the regression effect is good. Among them, $\alpha_{1}=-0.385, K_{1}=63605 . \alpha_{2}=-0.448, K_{2}$ $=50707 . \alpha_{3}=0.757$, and $K_{3}=34489$.

As shown in Table 6, the regression effect is good. The regression results of grey system and original data are different, but the positive and negative sign of their influence coefficient and the symbiotic relationship reflected are the same. Among them, $\alpha_{1}=0.212, K_{1}=75601, \alpha_{2}=0.297$, $K_{2}=43347, \alpha_{3}=0.407$, and $K_{3}=29156$. The competition system based on the Lotka-Volterra model is as follows:

$$
\left\{\begin{array}{l}
g_{1}(t)=0.212 N_{1}\left(1-\frac{N_{1}}{75601}+\frac{\beta_{12} N_{2}}{43347}+\frac{\beta_{13} N_{3}}{29156}\right) \\
g_{2}(t)=0.297 N_{2}\left(1-\frac{N_{2}}{43347}+\frac{\beta_{21} N_{1}}{75601}+\frac{\beta_{23} N_{3}}{29156}\right) \\
g_{3}(t)=0.407 N_{3}\left(1-\frac{N_{3}}{29156}+\frac{\beta_{31} N_{1}}{75601}+\frac{\beta_{32} N_{2}}{43347}\right)
\end{array} .\right.
$$


TABLE 4: Grey transformation error (MAPE).

\begin{tabular}{lcccc}
\hline Enterprise & $X^{(S)}(k)(\%)$ & $X^{(1)}(k)(\%)$ & $X^{(\mathrm{CG})}(k)(\%)$ & ARMA $(1,1)(\%)$ \\
\hline BMW & 14.970 & 7.647 & 5.786 & 25.933 \\
Benz & 12.908 & 10.586 & 6.629 & 20.720 \\
Audi & 17.627 & 11.735 & 8.400 & 30.735 \\
\hline
\end{tabular}

ARMA $(1,1)$

$$
\begin{array}{r}
40.00 \mathrm{X}(\mathrm{S})(\mathrm{k}) \\
30.00 \\
\bigcirc \\
\bigcirc 20.00
\end{array}
$$

$$
\begin{array}{r}
10.00 \\
\quad 0.00 \\
\hline
\end{array}
$$

\section{$\mathrm{X}(\mathrm{CG})(\mathrm{k})$}

$$
\begin{aligned}
& - \text { BMW } \\
& - \text { Benz } \\
& - \text { Audi }
\end{aligned}
$$

FIgURE 2: Comparison of error levels of four prediction models.

TABle 5: Competitive analysis of raw data based on the Lotka-Volterra model.

\begin{tabular}{lcccc}
\hline$N_{i}(t)$ & $\gamma_{\mathrm{i} 1}$ & $\gamma_{\mathrm{i} 2}$ & $\gamma_{\mathrm{i} 3}$ & $\gamma_{\mathrm{i} 4}$ \\
\hline$N_{1}(t)$ & $1.385(P \leq 0.001)$ & $-6.059 * 10^{-6}(P=0.176)$ & $5.580 * 10^{-6}(P=0.184)$ & $-7.078 * 10^{-6}(P=0.087)$ \\
$N_{2}(t)$ & $1.448(P \leq 0.001)$ & $-8.841 * 10^{-6}(P=0.145)$ & $7.653 * 10^{-6}(P=0.173)$ & $-7.316 * 10^{-6}(P=0.051)$ \\
$N_{3}(t)$ & $1.757(P \leq 0.001)$ & $-2.196 * 10^{-5}(P \leq 0.001)$ & $5.881 * 10^{-6}(P=0.225)$ & $3.164 * 10^{-6}(P=0.494)$ \\
\hline
\end{tabular}

TABLE 6: Competitive analysis of grey transformation data based on the Lotka-Volterra model.

\begin{tabular}{ccccc}
\hline$N_{i}(t)$ & $\gamma_{\mathrm{i} 1}$ & $\gamma_{\mathrm{i} 2}$ & $\gamma_{\mathrm{i} 3}$ & $\gamma_{\mathrm{i} 4}$ \\
\hline$N_{1}(t)$ & $1.212(P \leq 0.001)$ & $-2.814 * 10^{-6}(P=0.462)$ & $5.048 * 10^{-6}(P=0.174)$ & $-5.885 * 10^{-6}(P=0.057)$ \\
$N_{2}(t)$ & $1.297(P \leq 0.001)$ & $-6.856 * 10^{-6}(P=0.102)$ & $8.649 * 10^{-6}(P=0.037)$ & $-6.810 * 10^{-6}(P=0.017)$ \\
$N_{3}(t)$ & $1.407(P \leq 0.001)$ & $-1.398 * 10^{-5}(P \leq 0.001)$ & $4.073 * 10^{-6}(P=0.331)$ & $3.232 * 10^{-6}(P=0.420)$ \\
\hline
\end{tabular}

The equilibrium point of the three species symbiosis system is as follows:

$$
\left\{\begin{array}{l}
g_{1}(t)=0.212 N_{1}-\frac{0.212 N_{1}^{2}}{75601}+\frac{0.212 \beta_{12} N_{1} N_{2}}{43347}+\frac{0.212 \beta_{13} N_{1} N_{3}}{29156}=0 \\
g_{2}(t)=0.297 N_{2}-\frac{0.297 N_{2}^{2}}{43347}+\frac{0.297 \beta_{21} N_{1} N_{2}}{75601}+\frac{0.297 \beta_{23} N_{3} N_{2}}{29156}=0 \\
g_{3}(t)=0.407 N_{3}-\frac{0.407 N_{3}^{2}}{29156}+\frac{0.407 \beta_{31} N_{1} N_{3}}{75601}+\frac{0.407 \beta_{32} N_{2} N_{3}}{43347}=0
\end{array} .\right.
$$


The equivalent change can be obtained as follows:

$$
\begin{aligned}
& \Rightarrow\left\{\begin{array}{l}
N_{1}-\frac{N_{1}^{2}}{75601}+\frac{\beta_{12} N_{1} N_{2}}{43347}+\frac{\beta_{13} N_{1} N_{3}}{29156}=0 \\
N_{2}-\frac{N_{2}^{2}}{43347}+\frac{\beta_{21} N_{1} N_{2}}{75601}+\frac{\beta_{23} N_{3} N_{2}}{29156}=0 \\
N_{3}-\frac{N_{3}^{2}}{29156}+\frac{\beta_{31} N_{1} N_{3}}{75601}+\frac{\beta_{32} N_{2} N_{3}}{43347}=0
\end{array},\right. \\
& \int 1-\frac{N_{1}}{75601}+\frac{\beta_{12} N_{2}}{43347}+\frac{\beta_{13} N_{3}}{29156}=0 \\
& \Rightarrow\left\{1-\frac{N_{2}}{43347}+\frac{\beta_{21} N_{1}}{75601}+\frac{\beta_{23} N_{3}}{29156}=0,\right. \\
& 1-\frac{N_{3}}{29156}+\frac{\beta_{31} N_{1}}{75601}+\frac{\beta_{32} N_{2}}{43347}=0 \\
& \Rightarrow\left\{\begin{array}{l}
N_{1}=75601+\frac{75601 \beta_{12} N_{2}}{43347}+\frac{75601 \beta_{13} N_{3}}{29156} \\
N_{2}=43347+\frac{43347 \beta_{21} N_{1}}{75601}+\frac{43347 \beta_{23} N_{3}}{29156} \\
N_{3}=29156+\frac{29156 \beta_{31} N_{1}}{75601}+\frac{29156 \beta_{32} N_{2}}{43347}
\end{array}\right. \\
& \Rightarrow\left\{\begin{array}{l}
N_{1}=75601+1.744 \beta_{12} N_{2}+2.593 \beta_{13} N_{3} \\
N_{2}=43347+0.573 \beta_{21} N_{1}+1.487 \beta_{23} N_{3} \\
N_{3}=29156+0.386 \beta_{31} N_{1}+0.672 \beta_{32} N_{2}
\end{array}\right.
\end{aligned}
$$

By substituting the above symbiotic relationship into the MCGP model [28], the following results are obtained:

$$
\text { Objective function, } \operatorname{Min} \sum_{i=1}^{n}\left(d_{i}^{+}+d_{i}^{-}\right)+\sum_{i=1}^{n}\left(e_{i}^{+}+e_{i}^{-}\right)
$$

$$
\left\{\begin{array}{l}
\text { Constraints, } \\
g_{i}=f_{i}(x)+d_{i}^{-}-d_{i}^{+}, \quad i=1,2, \cdots, n \\
x \in X, X=\left\{x_{1}, x_{2}, \cdots, x_{m}\right\} \\
X \in F, \quad(\text { Fis the set of feasible solutions }) \\
g_{i, \max }=g_{i}+e_{i}^{-}-e_{i}^{+}, \quad i=1,2, \cdots, n \\
g_{i, \min } \leq g_{i}, g_{i} \leq g_{i, \max }, \quad i=1,2, \cdots, n \\
e_{i}^{+}, e_{i}^{-}, d_{i}^{+}, d_{i}^{-} \geq 0, \quad i=1,2, \cdots, n \\
N_{1}=75601+1.744 \beta_{12} N_{2}+2.593 \beta_{13} N_{3} \\
N_{2}=43347+0.573 \beta_{21} N_{1}+1.487 \beta_{23} N_{3} \\
N_{3}=29156+0.386 \beta_{31} N_{1}+0.672 \beta_{32} N_{2} \\
-1<\beta_{i j}<1, i=1,2,3 . j=1,2,3 . \\
\sum g_{i}=K,(\text { Kisthe sum of market capacity) }
\end{array}\right.
$$

The problem is solved using the LINGO [32] software. The results of $\beta_{\mathrm{ij}}$ value optimization of equilibrium symbiosis model are shown in Table 7 .

As shown in Table 7, with the expansion of market scale, more cooperation behaviors among populations are required. Cooperation behavior is comprehensive, and the intensity of cooperation is similar: the greater, the better.

2.5. Sensitivity Analysis of $\beta_{I j}$. This paper analyzes the change range of the model optimization results from the situation of $\beta_{\mathrm{ij}}$ with equal increase and simultaneous reverse change. The greater the change in the optimization result caused by the change of $\beta_{\mathrm{ij}}$ per unit, the stronger the sensitivity of $\beta_{\mathrm{ij}}$ in the model. In order to analyze the sensitivity of $\beta_{\mathrm{ij}}$ facilitate, here $\beta_{\mathrm{ij}}$ is set in the following table.

As shown in Table $8, \beta_{\mathrm{ij}}\left(\beta_{12}, \beta_{13} \beta_{21}, \beta_{23}, \beta_{31}\right.$, and $\left.\beta_{32}\right)$ has significant sensitivity in the model. When $\beta_{\mathrm{ij}}$ changes in the same direction and the same amount, the model optimizer can still run normally. The coordination change of $\beta_{\mathrm{ij}}$ significantly affects the model optimization results. The optimization result changes even more because of the coordination change of $\beta_{\mathrm{ij}}$. Therefore, $\beta_{\mathrm{ij}}$ is the most sensitive factor in the model.

\section{Results and Discussion}

In order to analyze the relationship between the variables in economic system and improve the performance of GM $(1,1)$ prediction, the three-dimensional grey Lotka-Volterra system is presented. The empirical results indicate that the numerical aspects of the grey transformation effect of the three-dimensional grey Lotka-Volterra's MAPE values are highly accurate. As compared with the GM $(1,1)$, the three-dimensional grey Lotka-Volterra model used in this study offers more accurate prediction performance. The combination of grey transformation data and three-dimensional Lotka-Volterra model can mine the relationship of populations in a certain system; accordingly, this method is suitable when the data is limited for predicting the relationship between competitive products. The empirical analysis of China's luxury car market fully demonstrates the effectiveness and adaptability of this method.

Compared with the traditional grey model [9-16], the three-dimensional grey Lotka-Volterra model can well describe the mechanism of population growth. Three-dimensional grey Lotka-Volterra model is more suitable for the research of social and economic ecosystem. Compared with the traditional Lotka-Volterra model [1-4], the threedimensional grey Lotka-Volterra model has higher accuracy. Compared with the grey Lotka-Volterra model $[5,17,18]$, the Grey-Logistic model [30], and the threedimensional Lotka-Volterra Model [31-34], the three-dimensional grey Lotka-Volterra model is more applicable. The case analysis based on three-dimensional grey Lotka-Volterra model fully demonstrates the accuracy and applicability of this method. Three-dimensional grey Lotka-Volterra model can effectively deal with the problem 
TABLE 7: Balanced point of symbiotic growth.

\begin{tabular}{|c|c|c|c|c|c|c|}
\hline Market size $(\mathrm{K})$ & 150000 & 180000 & 210000 & 240000 & 270000 & 300000 \\
\hline$\beta_{12}$ & 0.000 & 0.079 & 0.316 & 0.167 & 0.212 & 0.237 \\
\hline$\beta_{13}$ & 0.000 & 0.182 & 0.181 & 0.181 & 0.216 & 0.252 \\
\hline$\beta_{21}$ & 0.000 & 0.049 & 0.181 & 0.211 & 0.257 & 0.287 \\
\hline$\beta_{23}$ & 0.000 & 0.181 & 0.181 & 0.229 & 0.259 & 0.297 \\
\hline$\beta_{31}$ & 0.000 & 0.000 & 0.181 & 0.184 & 0.204 & 0.220 \\
\hline$\beta_{32}$ & 0.000 & 0.000 & 0.181 & 0.185 & 0.207 & 0.226 \\
\hline
\end{tabular}

TABLE 8: Sensitivity analysis of the proposed model by changing $\beta_{\mathrm{ij}}$.

\begin{tabular}{lccccccc}
\hline System state & 1 & 2 & 3 & 4 & 5 & 6 & 7 \\
\hline$\beta_{12}$ & 0 & 0.1 & 0.2 & 0.3 & 0.4 & 0.45 & 0.5 \\
$\beta_{13}$ & 0 & 0.1 & 0.2 & 0.3 & 0.4 & 0.45 \\
$\beta_{21}$ & 0 & 0.1 & 0.2 & 0.3 & 0.4 & 0.45 & 0.5 \\
$\beta_{23}$ & 0 & 0.1 & 0.2 & 0.3 & 0.4 & 0.45 & 0.45 \\
$\beta_{31}$ & 0 & 0.1 & 0.2 & 0.3 & 0.4 & 0.4 \\
$\beta_{32}$ & 0 & 0.1 & 0.2 & 0.3 & 0.4 & 0.5 \\
Market size (K) & 150000 & 185126 & 246826 & 370212 & 740263 & 1479880 & $1.69 \times 10^{9}$ \\
\hline
\end{tabular}

of insufficient data and missing data. When it is difficult to carry out regression analysis, the three-dimensional grey Lotka-Volterra model can be used to deal with it.

However, the model given in this study has its limitations in application, for example, (1) it does not reflect the life cycle mechanism of population growth, (2) the existing models cannot be used to analyze the symbiotic network, and (3) the model proposed in this paper cannot be used when studying the characteristics of data volatility. The above defects need to be remedied by future research.

\section{Conclusion}

The three-dimensional Lotka-Volterra model has been successfully built with the sales data of three local automobile enterprises in China. Based on Three Species System Analysis, it can be found that there is a symbiotic relationship among automobile enterprises and that the three species model can be adopted in analyzing the competition and cooperation among enterprises. Through balanced development of Symbiotic System Analysis, the result of symbiotic optimization under the equilibrium state of three populations is obtained. Equilibrium is possible in the growth of three populations; Three Species Evolutionary Analysis shows that cooperative strategy is better than competitive strategy and that this method is more practical in the analysis of enterprise competitive strategy. The findings reveal that this approach is feasible and effective to analyze competition, evolution, and balanced development of enterprise population. It is of great importance to study the relationships among enterprises as it is helpful for enterprises to make policies of strategy. Future studies could analyze the life cycle of competition among enterprises with three species Lotka-Volterra model.

This paper has a wide range of application scenarios. Grey three species Lotka-Volterra model can be used to analyze the symbiotic relationship between elements in natural and social systems. For example, (1) analysis of the relationship between competition and symbiosis of enterprise population; (2) analysis of symbiotic relationship among product populations within enterprises; (3) interaction analysis of investment portfolio; (3) prediction of input factors and output indicators in macro and regional economic systems; (4) analysis of symbiotic relationship of social ecosystem; and (5) symbiotic relationship analysis and population size prediction of biological populations in nature.

The research prospects of this paper are as follows: (1) the life cycle mechanism of population growth is introduced into the grey three species Lotka-Volterra model; (2) symbiotic network analysis models and methods can be developed based on existing models; and (3) the population dynamics method proposed in this paper can be combined with the machine learning method considering Chaotic Systems dynamics [35-37]. The new compound method can make full use of the new progress of machine learning research to transform population dynamics.

\section{Data Availability}

The experimental data used to support the findings of this study are included within the article.

\section{Conflicts of Interest}

The authors declare that they have no conflicts of interest.

\section{Acknowledgments}

This work was supported by the National Social Science Foundation of China (no. 20BGL203). 


\section{References}

[1] G. Gandolfo, "Giuseppe palomba and the lotka-volterra equations," Rendiconti Lincei, vol. 19, no. 4, pp. 347-357, 2008.

[2] V. B. Kreng and H. T. Wang, "The competition and equilibrium analysis of LCD TV and PDP TV," Technological Forecasting and Social Change, vol. 78, no. 3, pp. 448-457, 2011.

[3] S.-Y. Chiang, "An application of Lotka-Volterra model to Taiwan's transition from $200 \mathrm{~mm}$ to $300 \mathrm{~mm}$ silicon wafers," Technological Forecasting and Social Change, vol. 79, no. 2, pp. 383-392, 2012.

[4] P. Jiang, X. B. Yan, and L. Y. Wang, "A viral product diffusion model to forecast the market performance of products," Discrete Dynamics in Nature and Society, vol. 2017, Article ID 9121032, 10 pages, 2017.

[5] H.-T. Wang and T.-C. Wang, "Application of the grey LotkaVolterra model to forecast the diffusion and competition analysis of the TV and smartphone industries," Technological Forecasting and Social Change, vol. 106, pp. 37-44, 2016.

[6] T. Wei and T. T. Liu, "Evolution of high-value patents in reverse innovation: focus on Chinese local enterprises," Mathematical Problems in Engineering, vol. 2020, Article ID 8127096, 13 pages, 2020.

[7] G. M Lopes and J. F. Fontanari, "Influence of technological progress and renewability on the sustainability of ecosystem engineers populations," Mathematical Biosciences and Engineering, vol. 16, no. 5, pp. 3450-3464, 2019.

[8] B. Meng, H. Kuang, E. Niu, J. Li, and Z. Li, "Research on the transformation path of the green intelligent port: outlining the perspective of the evolutionary game Government-PortThird-Party organization," Sustainability, vol. 12, no. 19, p. 8072, 2020.

[9] J. L. Deng, "Introduction to Grey system theory," Journal of Grey System, vol. 1, no. 1, pp. 191-243, 1989.

[10] S. F. Liu and Y. Lin, Grey Systems: Theory and Practical Applications, Springer-Verlag London Ltd., London, 2010.

[11] Y.-S. Chen and B.-Y. Chen, "Applying DEA, MPI, and grey model to explore the operation performance of the Taiwanese wafer fabrication industry," Technological Forecasting and Social Change, vol. 78, no. 3, pp. 536-546, 2011.

[12] C. T. Lin and S. Y. Yang, "Forecast of the output value of Taiwan's opto-electronics industry using the Grey forecasting model," Technological Forecasting and Social Change, vol. 70, no. No. 2, pp. 177-186, 2003.

[13] S.-C. Lee and L.-H. Shih, "Forecasting of electricity costs based on an enhanced gray-based learning model: a case study of renewable energy in Taiwan," Technological Forecasting and Social Change, vol. 78, no. 7, pp. 1242-1253, 2011.

[14] L.-C. Hsu, "Applying the Grey prediction model to the global integrated circuit industry," Technological Forecasting and Social Change, vol. 70, no. 6, pp. 563-574, 2003.

[15] Y.-Y. Tai, J.-Y. Lin, M.-S. Chen, and M.-C. Lin, "A grey decision and prediction model for investment in the core competitiveness of product development," Technological Forecasting and Social Change, vol. 78, no. 7, pp. 1254-1267, 2011.

[16] M. Mao and E. C. Chirwa, "Application of grey model GM(1, 1) to vehicle fatality risk estimation," Technological Forecasting and Social Change, vol. 73, no. 5, pp. 588-605, 2006.

[17] L. Wu, S. Liu, and Y. Wang, "Grey Lotka-Volterra model and its application," Technological Forecasting and Social Change, vol. 79, no. 9, pp. 1720-1730, 2012.
[18] S. Mao, M. Zhu, X. Wang, and X. Xiao, "Grey-Lotka-Volterra model for the competition and cooperation between thirdparty online payment systems and online banking in China," Applied Soft Computing, vol. 95, Article ID 106501, 2020.

[19] H.-C. Lee and C.-T. Chang, "Comparative analysis of MCDM methods for ranking renewable energy sources in Taiwan," Renewable and Sustainable Energy Reviews, vol. 92, pp. 883896, 2018.

[20] O. Mohsen and N. Fereshteh, "An extended VIKOR method based on entropy measure for the failure modes risk assessment-a case study of the geothermal power plant (GPP)," Safety Science, vol. 92, pp. 160-172, 2017.

[21] R. Shad, M. Khorrami, and M. Ghaemi, "Developing an Iranian green building assessment tool using decision making methods and geographical information system: case study in Mashhad city," Renewable and Sustainable Energy Reviews, vol. 67, pp. 324-340, 2017.

[22] A. Hafezalkotob and A. Hafezalkotob, "Extended MULTIMOORA method based on Shannon entropy weight for materials selection," Journal of Industrial Engineering International, vol. 12, no. 1, pp. 1-13, 2016.

[23] F. H. Lotfi and R. Fallahnejad, "Imprecise shannon's entropy and multi attribute decision making," Entropy, vol. 12, no. 1, pp. 53-62, 2010.

[24] V. Volterra, "Fluctuations in the abundance of a species considered Mathematically1," Nature, vol. 118, no. 2972, pp. 558-560, 1926.

[25] G. L. Zhang, A. Daniel, and V. M. Adams, "Technology evolution prediction using Lotka-Volterra Equations," Journal of Mechanical Design, vol. 140, no. 6, pp. 61-101, 2018.

[26] T. Modis, "Technological forecasting at the stock market," Technological Forecasting and Social Change, vol. 62, no. 3, pp. 173-202, 1999.

[27] C.-T. Chang, C.-Y. Ku, and H.-P. Ho, "Fuzzy multi-choice goal programming for supplier selection," International Journal of Operations Research and Information Systems, vol. 1, no. 3, pp. 28-52, 2010.

[28] S.-Y. Wang, W.-M. Chen, and Y. Liu, "Collaborative product portfolio design based on the approach of multi choice goal programming," Mathematical Problems in Engineering, vol. 2021, Article ID 6678533, 16 pages, 2021.

[29] C.-T. Chang, "Multi-choice goal programming," Omega, vol. 35, no. 4, pp. 389-396, 2007.

[30] X.-L. Wu, S.-Y. Wang, and G.-Y. Xu, "Compound grey -LogisticModel and its application," Mathematical Problems In Engineering, vol. 2021, Article ID 5588798, 13 pages, 2021.

[31] S.-Y. Wang, W.-M. Chen, and X.-L. Wu, "Competition analysis on industry populations based on a three-dimensional lotka-volterra model," Discrete Dynamics in Nature and Society, vol. 2021, Article ID 9935127, 15 pages, 2021.

[32] L. Schrage, LINGO Release 8.0, LINDO System, Inc, Chicago, IL, USA, 2002.

[33] F.-D. Dong, W.-T. Li, and J.-B. Wang, "Propagation dynamics in a three-species competition model with nonlocal anisotropic dispersal," Nonlinear Analysis: Real World Applications, vol. 48, pp. 232-266, 2019.

[34] Q. Liu, S. Liu, and K.-Y. Lam, "Stacked invasion waves in a competition-diffusion model with three species," Journal of Differential Equations, vol. 271, pp. 665-718, 2021.

[35] J. Pathak, Z. Lu, B. Hunt, M. Girvan, and E. Ott, "Using machine learning to replicate chaotic attractors and calculate Lyapunov exponents from data," Chaos, vol. 27, Article ID 121102, 2017. 
[36] Z. Lu, J. Pathak, B. Hunt, M. Girvan, R. Brockett, and E. Ott, "Reservoir observers: model-free inference of unmeasured variables in chaotic systems," Chaos: An Interdisciplinary Journal of Nonlinear Science, vol. 27, no. 4, Article ID 041102, 2017.

[37] J. Pathak, B. Hunt, M. Girvan, Z. Lu, and E. Ott, "Model-Free prediction of large spatiotemporally chaotic systems from data: a reservoir computing approach," Physical Review Letters, vol. 120, no. 2, 2018. 